\title{
Pricing of Some Exotic Options with NIG-Lévy Input
}

\author{
Sebastian Rasmus ${ }^{1}$, Søren Asmussen ${ }^{2}$, and Magnus Wiktorsson ${ }^{1}$ \\ 1 Center for Mathematical Sciences, University of Lund, \\ Box 118, 22100 Lund, Sweden \\ \{rasmus, magnusw\}@maths. Ith. se \\ 2 Theoretical Statistics, University of Aarhus, \\ Ny Munkegade, 8000 Aarhus C, Denmark \\ asmus@imf .au.dk
}

\begin{abstract}
We study the problem of pricing barrier options and Russian options driven by exponential NIG Lévy processes by simulation. Simulating at a discrete grid creates a systematic bias because the minimum and maximum in between grid points is neglected. The proposed solution is to simulate the large jumps only and use a Brownian approximation for the rest combined with explicit formulas for Brownian minima and maxima.
\end{abstract}

\section{Introduction}

Models for financial data different from the classical Black-Scholes (B\&S) geometric Brownian motion (BM) have recently received considerable attention, see for instance Boyarchenko \& Levendorskii [8], Chan [9] and Schoutens [16]. The motivation is to a large extent statistical since many log-returns show deviations from normality. The most popular alternative is Lévy process models, and in particular the NIG model (see Barndorff Nielsen [6]) has become popular because of its flexibility.

The consequence for option prices are multiple. One is non-uniqueness of risk-neutral measures, another that the wealth of explicit formulas for BM in the $\mathrm{B} \& \mathrm{~S}$ world is no longer available.

For simple options like Europeans call, only marginal distributions are required and pricing may be done by numerical integration. For slightly more complicated examples like Asian options, one may just simulate along a discrete grid. Numerical studies for such problems and comparison with the B\&S prices have been carried out for the NIG case in Albrecher \& Predota 2]. The present paper continues this work by studying some options (barrier options and Russian options) where the computational aspect is more complicated.

\section{Lévy Processes}

A Lévy process is a stochastically continuous process with stationary and independent increments. This implies the existence of a characteristic exponent $(\kappa(\xi))$ which form is given by the Lévy triplet $(a, \sigma, \nu)$ as follows, 


$$
\mathrm{E}\left[e^{i \xi X_{t}}\right]=e^{t \kappa(i \xi)}=\exp \left\{t\left(i a \xi-\frac{\sigma^{2} \xi^{2}}{2}+\int_{\mathbb{R}}\left(e^{i \xi x}-1-i \xi x \mathbf{1}_{\{|x|<1\}}\right) \nu(d x)\right)\right\}
$$

where $a, \xi \in \mathbb{R}, \sigma \in \mathbb{R}_{+}$and $\nu$ a measure on $\mathbb{R} \backslash\{0\}$ which satisfies $\int_{\mathbb{R}}\left(x^{2} \wedge 1\right) \nu(d x)<\infty$. The finite and infinite variation case refer to the integral, $\int_{|x|<1}|x| \nu(d x)$ being finite or infinite. See Bertoin [7] and Sato [13 for more details.

\subsection{The Esscher Transform}

If the Lévy process $X_{t}$ is a BM or a Poisson process the stock price model based on

$$
S_{t}=S_{0} e^{X_{t}}
$$

is complete. In all other cases the model is incomplete (see Chan 9] and Cherny \& Shiryaev [10]) and a there are many martingale measures $\mathbb{Q}$ making $X_{t}$ a Lévy process and satisfying

$$
e^{r t}=\mathrm{E}^{\mathbb{Q}}\left[e^{X_{t}}\right]=\exp \left\{t \kappa^{\mathbb{Q}}(1)\right\}
$$

where $\kappa^{\mathbb{Q}}$ is the characteristic exponent of $X_{t}$ under $\mathbb{Q}$. Within the class of exponentially tilted Lévy processes, i.e. with $\kappa^{\mathbb{Q}}$ of the form

$$
\kappa^{\mathbb{Q}}(x)=\kappa(x+\theta)-\kappa(\theta)
$$

the equation $\kappa^{\mathbb{Q}}(1)=r$ has either a unique solution $\theta_{0}$ (this is the typical situation for light tails and the corresponding $\kappa^{\mathbb{Q}}$ is called the Esscher transform) or none (typical for heavy tails).

\subsection{The NIG Lévy Process}

The $N I G(\alpha, \beta, \mu, \delta)$ distribution, defined for $\alpha, \delta \geq 0,|\beta| \leq \alpha, \mu \in \mathbb{R}$ has density

$$
f(x)=\frac{\alpha \delta}{\pi} \frac{K_{1}\left(\alpha \sqrt{\delta^{2}+(x-\mu)^{2}}\right)}{\sqrt{\delta^{2}+(x-\mu)^{2}}} \exp \{\delta \gamma+\beta(x-\mu)\}
$$

where $\gamma^{2}=\alpha^{2}-\beta^{2}$ and $K_{1}$ is the modified Bessel function of the third kind. The $N I G$ Lévy process is a Lévy process such that $X_{t}$ has a $N I G(\alpha, \beta, \mu t, \delta t)$ distribution. It is a pure jump process and its Lévy measure has density

$$
\nu(x ; \alpha, \beta, \delta)=\frac{\delta \alpha}{\pi|x|} \exp \{\beta x\} K_{1}(\alpha|x|)
$$

With this the characteristic exponent is given by

$$
\kappa(\xi)=\mu \xi+\delta\left[\left(\alpha^{2}-\beta^{2}\right)^{1 / 2}-\left(\alpha^{2}-(\beta+\xi)^{2}\right)^{1 / 2}\right]
$$

It is easy to see ([2]) that under the Esscher measure $X_{t}$ is still a $N I G$ Lévy process, with the same $\alpha, \mu, \delta$ but $\beta$ replaced by

$$
\beta+\theta_{0}=-\frac{1}{2}+\sqrt{\frac{(\mu-r)^{2}}{\delta^{2}+(\mu-r)^{2}} \alpha^{2}-\frac{(\mu-r)^{2}}{4 \delta^{2}}}
$$




\section{Simulating Jumps from the Lévy Measure}

The Lévy measure $\nu$ contains all information about the jumps. If its total mass $\lambda$ over $\mathbb{R}$ is finite, the jump times form a Poisson process with intensity $\lambda$ and the jump size distribution is $g(d x)=\nu(d x) / \lambda$. So, if $g$ can be simulated the path of a Lévy process can be generated as a compound Poisson process: first a random time grid from an $\operatorname{Exp}(\lambda)$ distribution is drawn and at each grid point we draw a jump from $g$.

Since the Lévy measure is allowed to have infinite mass in an open interval containing zero the compound Poisson method may break down. In Asmussen \& Rosinski [4] it is shown (under certain conditions valid for the NIG case) that jumps smaller than $\epsilon>0$ may be approximated by a BM with drift and variance given by,

$$
m(\epsilon)=-\int_{\epsilon<|x|<1} x \nu(d x), \quad v^{2}(\epsilon)=\int_{|x|<\epsilon} x^{2} \nu(d x)
$$

So, the general idea is to use the compound Poisson process method above for jumps bounded away from zero, say larger than $\epsilon\left(J_{>\epsilon}\right)$. To be explicit we use the notation $\lambda_{\epsilon}$ and $g_{\epsilon}$. The rest of the jumps, in $[-\epsilon, \epsilon]\left(J_{<\epsilon}\right)$, is then approximated by a BM with drift $m(\epsilon)$ and variance $v^{2}(\epsilon)$.

\subsection{Reflecting the Lévy Process}

For the Russian option we are interested in the reflected (at the supremum) version $R_{t}$ of the Lévy process $X_{t}$ and the hitting time $\tau_{k}$ of the barrier $k$, i.e.

$$
R_{t}=\sup _{0 \leq s \leq t} X_{s}-X_{t} \quad \tau_{k}=\inf \left\{t>0: R_{t} \geq k\right\}
$$

In the infinite variation case the small jump approximation is particularly important. Consider the simulation of the running maximum $M_{t}^{X}=\sup _{0 \leq s \leq t} X_{s}$. On the random grid generated by the compound Poisson process of the large jumps $J_{>\epsilon}$ a maximum between the grid points may be missed. Since there is an explicit formula for the joint distribution of the BM and its maximum (see for instance Asmusen, Glynn \& Pitman [3] Algorithm B) the small jump approximation make it possible to compensate for this bias. Note also that since $J_{<\epsilon}$ may be approximated by the well known BM, this compensation is computable.

This aspect becomes even more important simulating $\tau_{k}$. Here we need the running maximum $M_{t}^{R}$ of $R_{t}$, which in turn needs $M_{t}^{X}$. To update them both over a grid interval we need the joint distribution the infimum and supremum of a BM over a compact interval. This is unknown to us and we do the following approximation. As time evolve $M_{t}^{X}$ and $M_{t}^{R}$ are updated independently over each of the exponential intervals between the jumps. This should be a very close approximation since the probability that they both are updated simultaneously over such an interval is negligible: if $X_{t}$ is close to $M_{t}^{X}, R_{t}$ is close to 0 , i.e. far from $M_{t}^{R}$ and vice versa. 


\subsection{The NIG Case}

In the paper Asmussen \& Rosinski [4] it is explicitly shown that the small jump approximation is valid. So, the small jumps are given by the equations (3) and we turn directly to the large jumps.

Using the the asymptote of the Bessel function $K_{1}$ in Abramowits \& Stegun [1], the corresponding asymptote for the density of the NIG Lévy measure is,

$$
\begin{array}{ll}
\nu(x)=\frac{\delta \alpha}{\pi|x|} \exp \{\beta x\} K_{1}(\alpha|x|) \sim \frac{C_{2}}{x^{2}} \quad x \downarrow 0 \quad \text { or } x \uparrow 0 \\
\nu(x)=\frac{\delta \alpha}{\pi|x|} \exp \{\beta x\} K_{1}(\alpha|x|) \sim \frac{C_{1}}{|x|^{3 / 2}} \exp \{\beta x-\alpha|x|\} & x \rightarrow \pm \infty
\end{array}
$$

These asymptotes give a hint of how to sample from $g_{\epsilon}(x)$. Consider first only $x>\epsilon$ (the case $x<-\epsilon$ is analogous)

In this paper we use an Accept and Reject method with a mixed density $f(x)$. For $x \in[\epsilon, \omega]$ we use a quadratic density $f_{q}(x)$ and in the tail, $x>\omega$, an exponential density $f_{e}(x)$ is used.

$$
\begin{aligned}
& f_{q}(x)=\left(\frac{1}{\epsilon}-\frac{1}{\omega}\right)^{-1} \frac{1}{x^{2}} \quad x \in[\epsilon, \omega] \\
& f_{e}(x)=(\alpha-\beta) \exp \{-(\alpha-\beta)(x-\omega)\} \quad x>\omega
\end{aligned}
$$

This choice majorises the density $g_{\epsilon}$ for the asymptotes $x \downarrow 0$ and $x \rightarrow \infty$.

\section{Derivative Prices}

To evaluate this simulation algorithm we price some derivatives and see how they differ from the B\&S prices. The derivatives chosen are listed below and in both cases there exists a closed form pricing formula from the B\&S case.

The Barrier Option. We consider the up-in Barrier option (with barrier $H$ ) on the European call option (with strike $K$ ). The payoff function is,

$$
\Phi^{B}(T)=\left(S_{T}-K\right) \mathbf{1}\left\{S_{T}>K, M_{T}^{S}>H\right\}
$$

where $M_{t}^{S}$ is the running maximum of $S_{s}$ up to time $t$.

The Russian Option. The Russian option is a contract that gives the holder the right to exercise at any ((a.s) finite) stopping time $\tau$ yielding the payoff

$$
\Phi^{R}(\tau)=e^{-\eta \tau} \max \left\{M_{0}, \sup _{0 \leq u \leq \tau} S_{u}\right\}, \quad M_{0} \geq S_{0}, \eta>0
$$

where $S_{t}$ is the underlying asset, $M_{0}$ is the starting maximum and $\eta$ is the punish factor. In this paper we use $M_{0}=S_{0}$ and vary $\eta$. 
Some references for the B\&S model are Shepp \& Shiryaev [14] \& [15] and Duffie \& Harrison [11]. For the case of a geometric Lévy process see Asmussen, Avram \& Pistorius [5]. The computation involve the first passage function,

$$
v_{k}(y)=\mathrm{E}_{y}\left[\exp \left\{-\eta \tau_{k}+R_{\tau_{k}}\right\}\right]
$$

which is maximized over $k$ and $R_{t}$ and $\tau_{k}$ from equation (4).

\section{$5 \quad$ Results}

We present two studies using this algorithm. The first is an evaluation how important the BM compensation is. Secondly, there is a convergence of the NIG towards the B\&S prices. We try to evaluate the speed for different derivatives.

\subsection{Data}

We use the estimations of the Dresdner Bank stock in the paper by Rydberg [12. The parameters are,

$$
(\alpha, \beta, \mu, \delta)=(68.28,1.81,0,0.01)
$$

Under the Esscher transform $\beta=0.8653$ and to compare with the B\&S prices we need $\operatorname{Var}\left(X_{1}\right)=1.46610^{-4}$. The following table shows how the jump intensity $\lambda(\epsilon)$ (which may be identified with the computational effort) varies with the truncation at $\epsilon$.

Table 1. Intensities $\lambda_{\epsilon}$ for different truncations $\epsilon$

\begin{tabular}{|l|c|c|c|c|c|c|c|}
\hline$\epsilon$ & $10^{-2}$ & $2 \cdot 10^{-3}$ & $10^{-3}$ & $2 \cdot 10^{-4}$ & $10^{-4}$ & $2 \cdot 10^{-5}$ & $10^{-5}$ \\
\hline$\lambda(\epsilon)$ & 0.25636 & 2.6075 & 5.74728 & 31.1660 & 62.989 & 317.63 & 635.94 \\
\hline
\end{tabular}

\subsection{Grid Discretization}

In this section we show some results indicating how well the grid compensation works. The barrier option depends on the hitting of a barrier. In this case it is only the asset that should hit a barrier. In the Russian option case the reflected process should hit the barrier, which actually involve two different running maximums, i.e. updating $M^{X}$ to get $R$ and $M^{R}$ to update $\tau_{k}$.

Barrier option. Here the time to maturity $T=10$ and the barrier level $H=1.055$ is fixed and we consider different strike prices $K$ and grid intensities, that is we vary $\epsilon$. We present $0.95 \%$ confidence intervals where $0.9 \mathrm{U}$ means upper bound for $K=0.9$ for instance. The number of simulations are $2 \cdot 10^{5}$. 
The conclusion we make is that the truncation works well for $\epsilon<10^{-3}$. Without compensation however, it looks like we need $\epsilon<2 \cdot 10^{-4}$ that is, about 6 times as much computer time, c.f. the Table 1 above. Smaller $\epsilon$ give similar confidence bands as $\epsilon=10^{-4}$ both with or without grid compensation.

Table 2. Barrier option prices for different $\epsilon$ with grid compensation

\begin{tabular}{|c||c|c||c|c||c|c||c|c||}
\hline$(\epsilon, K)$ & $0.9 \mathrm{~L}$ & $0.9 \mathrm{U}$ & $0.95 \mathrm{~L}$ & $0.95 \mathrm{U}$ & $1 \mathrm{~L}$ & $1 \mathrm{U}$ & $1.05 \mathrm{~L}$ & $1.05 \mathrm{U}$ \\
\hline \hline$\epsilon=10^{-2}$ & 0.0274 & 0.0280 & 0.0189 & 0.0193 & 0.0104 & 0.0106 & 0.00325 & 0.00335 \\
\hline$\epsilon=2 \cdot 10^{-3}$ & 0.0221 & 0.0226 & 0.0152 & 0.0156 & 0.00840 & 0.00860 & 0.00225 & 0.00233 \\
\hline$\epsilon=10^{-3}$ & 0.0218 & 0.0223 & 0.0151 & 0.0154 & 0.00829 & 0.00849 & 0.00217 & 0.00226 \\
\hline$\epsilon=2 \cdot 10^{-4}$ & 0.0216 & 0.0221 & 0.0149 & 0.0152 & 0.00822 & 0.00842 & 0.00214 & 0.00222 \\
\hline$\epsilon=10^{-4}$ & 0.0217 & 0.0221 & 0.0149 & 0.0153 & 0.00822 & 0.00842 & 0.00215 & 0.00223 \\
\hline
\end{tabular}

Table 3. Barrier option prices for different $\epsilon$ without grid compensation

\begin{tabular}{|c||c|c||c|c||c|c||c|c||}
\hline$(\epsilon, K)$ & $0.9 \mathrm{~L}$ & $0.9 \mathrm{U}$ & $0.95 \mathrm{~L}$ & $0.95 \mathrm{U}$ & $1 \mathrm{~L}$ & $1 \mathrm{U}$ & $1.05 \mathrm{~L}$ & $1.05 \mathrm{U}$ \\
\hline \hline$\epsilon=10^{-2}$ & 0.0141 & 0.0145 & 0.00991 & 0.0102 & 0.00579 & 0.00599 & 0.00216 & 0.00225 \\
\hline$\epsilon=2 \cdot 10^{-3}$ & 0.0205 & 0.0210 & 0.0142 & 0.0145 & 0.00792 & 0.00812 & 0.00222 & 0.00230 \\
\hline$\epsilon=10^{-3}$ & 0.0211 & 0.0216 & 0.0146 & 0.0149 & 0.00808 & 0.00828 & 0.00216 & 0.00225 \\
\hline$\epsilon=2 \cdot 10^{-4}$ & 0.0215 & 0.0220 & 0.0148 & 0.0152 & 0.00818 & 0.00838 & 0.00214 & 0.00222 \\
\hline$\epsilon=10^{-4}$ & 0.0216 & 0.0221 & 0.0149 & 0.0152 & 0.00820 & 0.0084 & 0.00215 & 0.00223 \\
\hline
\end{tabular}

Russian option. Here we fix the punish level $\eta=3 \cdot 10^{-3}$ and vary $\epsilon$, which implies $\tau_{k} \sim 10$. Again we present $0.95 \%$ confidence intervals for the case with compensation and the next two without compensation.

Again we conclude that the truncation works well for $\epsilon<10^{-3}$ in the compensated case. Without compensation we need $\epsilon<2 \cdot 10^{-4}$.

Table 4. Russian option prices for different $\epsilon$

\begin{tabular}{|c|c|c|c|c|}
\hline$\epsilon$ & Comp-Low & Comp-Up & No Comp-Low & No Comp-Up \\
\hline \hline$\epsilon=10^{-2}$ & 1.0229 & 1.0242 & 1.0117 & 1.0170 \\
\hline$\epsilon=2 \cdot 10^{-3}$ & 1.0080 & 1.0084 & 1.0059 & 1.0062 \\
\hline$\epsilon=10^{-3}$ & 1.0074 & 1.0077 & 1.0064 & 1.0068 \\
\hline$\epsilon=2 \cdot 10^{-4}$ & 1.0074 & 1.0077 & 1.0072 & 1.0076 \\
\hline$\epsilon=10^{-4}$ & 1.0075 & 1.0078 & 1.0074 & 1.0078 \\
\hline
\end{tabular}




\subsection{Convergence to B\&S Prices}

Since the variance of the $N I G$ variable is finite, Donsker's Theorem

$$
\left\{\frac{X_{t T}-a t T}{\sqrt{b^{2} T}}\right\}_{t \geq 0} \rightarrow B_{t} \text { as } T \rightarrow \infty
$$

suggests that NIG and the B\&S prices are not too far if the time horizon is large. In this section we give some examples quantifying this.

Barrier option. The closeness measure used in the Barrier option case is

$$
Q(K)=\frac{\Pi^{B \& S}(K)}{\Pi^{N I G}(K)}
$$

Since we assume that $S_{0}=1, K$ represent the moneyness in the option.

Note that for $T=2$ is $Q<1$ for all $K$ and close to 1 at $T=4$ for $K$ small. For larger $T$ will $Q$ grow (see $T=30$ ) and then tend to 1 for all $K \in[0.9,1.05]$ $(T=80)$ as we expected.
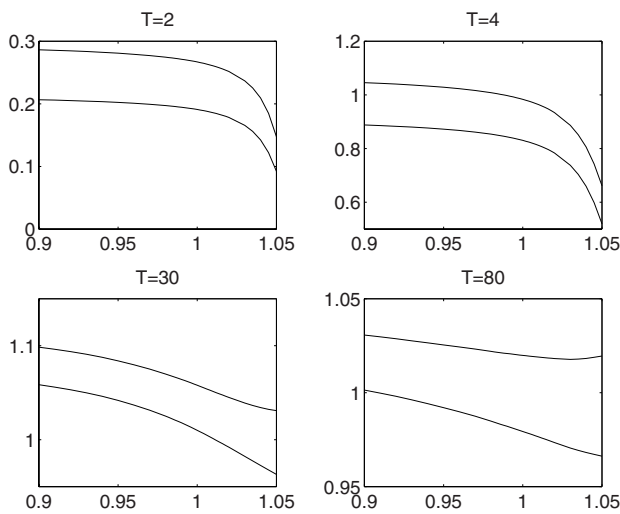

Fig. 1. Confidence intervals for $Q$ against $K$ for different maturities $T$

Russian option. In the B\&S case there is a closed form solution but in the NIG case we present a $95 \%$ confidence interval.

The conclusion here is that the relative price quotient above $S_{0}=1$ decreases for smaller punish factor, that is longer time horizon. 


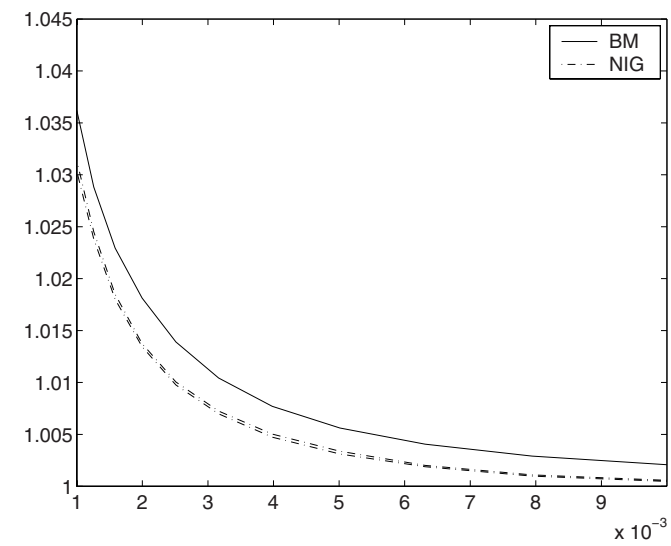

Fig. 2. Price of Russian option against punish factor

\section{References}

1. Abramowitz, M. \& Stegun, I.A. (editors) (1972). Handbook of Mathematical Functions. Dover, New York.

2. Albrecher, H. \& Predota, M. (2004). On Asian option pricing for NIG Lévy processes, Journal of Comp. and Appl. Math.

3. Asmusen, S., Glynn, P. \& Pitman, J. (1995). Discretization error in simulating of one-dimensional reflecting Brownian motion. Ann. of Appl. Prob. 5, 875-896.

4. Asmussen, S. \& Rosinski, J. (2001). Approximation of small jumps of Lévy processes with a view towards simulation. J. Appl. Prob. 38, 482-493.

5. Asmussen, S., Avram, F. \& Pistorius, M. (2004). Russian and American put options under exponential phase-type Lévy motion, Stoch. Proc. Appl. 109, 79-111.

6. Barndorff-Nielsen, O. (1998). Processes of normal inverse gaussian type. Finance and Stochastics. 2, 41-68.

7. Bertoin, J. (1996). Lévy Processes, Cambridge University Press.

8. Boyarchenko, S. \& Levendorskiï, S. (2002). Non Gaussian Merton-Black-Scholes Theory, World Scientific.

9. Chan, T. (1999). Pricing contingent claims with Lévy processes. Ann. Appl. Prob. 9, 502-528.

10. Cherny, A.S. \& Shiryaev, A.N. (2001). Change of time and measure for Lévy processes. MaPhySto Lecture Notes. 13 (Available on http://www.maphysto.dk/).

11. Duffie, D. \& Harrison, M. (1993). Arbitrage pricing of Russian options and perpetual Look back options. Ann. of Appl. Prob. 3, 641-651.

12. Rydberg, T.H (1997). The Normal Inverse Gaussian process: simulation and approximation. Stoch. Models. 13, 887-910.

13. Sato, K-I. (1999). Lévy Processes and Infinitely Divisible Distributions, Cambridge University Press.

14. Shepp, L. \& Shiryaev, A.N. (1993). The Russian option: reduced regret. Ann. of Appl. Prob. 3, 631-640.

15. Shepp, L. \& Shiryaev, A.N. (1994). A new look at the "Russian option". Theory Prob. Appl. 39, 103-119.

16. Schoutens, W. (2003). Lévy Processes in Finance, Wiley. 